\title{
A Survey of the Polytrauma Outpatient Population: Technological Capabilities and Potential for Telerehabilitation
}

\author{
Kerry-Ann Stewart ${ }^{1}$, Max Gray ${ }^{2}$, Joyce Chung ${ }^{2}$, Odette Harris ${ }^{3}$ \\ 1. Department of Veterans Affairs, VA Palo Alto Health Care System; Stanford University School of \\ Medicine 2. Department of Veterans Affairs, VA Palo Alto Health Care System 3. Department of \\ Neurosurgery, Stanford University School of Medicine
}

$\square$ Corresponding author: Max Gray, max.gray@va.gov

Disclosures can be found in Additional Information at the end of the article

\section{Abstract}

Background: Rapid technological advances in personal communication devices have provided new opportunities for their use to provide care, education and support to patients and families in remote locations via telerehabilitation applications. Studies indicate that individuals with brain injury are interested in utilizing internet telerehabilitation services. However, there is minimal data regarding whether these patients have access to the necessary telecommunication devices. As new communication technologies develop, we need a clear understanding of the acceptance of those technologies by the intended service population. Our unique access to the TBI outpatient population allows this study to fill the knowledge gap by exploring the availability of advanced telecommunication devices within that cohort.

Methods: A prospective cohort of 70 polytrauma outpatient clinic patients with a history of traumatic brain injury was surveyed via telephone. Respondents were living in the community at the time the survey was conducted. The survey was administered in a structured telephone interview format and analyzed qualitatively.

Results: In this cohort, 94\% reported ownership and daily use of cell phones, $41 \%$ had a Smartphone, 97\% reported at least weekly use of a computer, and $81 \%$ indicated at least once daily internet use on either a computer or a Smartphone. Cost and lack of necessity were endorsed as barriers to ownership and use of these devices.

Conclusions: These results suggest that a significant proportion of individuals with documented TBI have access to advanced and evolving communication technologies that can potentially be utilized for telerehabilitation.

Received 12/21/2012

Review began 12/22/2012

Published 01/23/2013

\section{C) Copyright 2013}

Stewart et al. This is an open access article distributed under the terms of the Creative Commons Attribution License CC-BY 3.0., which permits unrestricted use, distribution, and reproduction in any medium, provided the original author and source are credited.
Categories: Physical Medicine \& Rehabilitation, Miscellaneous, Neurosurgery Keywords: telemedicine, telerehabilitation, traumatic brain injury, polytrauma, neurosurgery

\section{Introduction}

Traumatic brain injury (TBI) is an immense public health problem affecting over one million people in the United States annually [1]. The conflicts in Afghanistan and Iraq have resulted in an unprecedented increase in the number of returning military personnel with TBI. It is estimated that at least $22 \%$ of wounded soldiers evacuated from the Operation Iraqi Freedom (OIF) and Operation Enduring Freedom (OEF) conflicts sustained a TBI [2]. According to the 
Department of Defense, between the years 2000 and 2010, a total of 195,547 service members within the US military have been diagnosed with TBI [3]. Thus, TBI is often referred to as the "signature injury" of troops serving in the OIF/OEF conflicts [4].

To address the care of this specialized patient cohort, the Polytrauma System of Care (PSC) continuum was established in 2005 with Veterans Affairs Palo Alto Health Care System (VAPAHCS) being designated as one of four Polytrauma Centers nationwide. This designation includes providing services for in-patients through polytrauma rehabilitation center (PRC) and polytrauma transitional rehabilitation programs (PTRP), and for outpatients through polytrauma network site (PNS) clinics. The system operates facilities that include three inpatient sites, Palo Alto, Menlo Park and Livermore. It also operates six community based outpatient clinics (CBOCs), Capitola, Modesto, Monterey, San Jose, Sonora, and Stockton to cover its wide catchment area encompassing 10 Northern California counties and over 13,500 square miles. Much of this catchment area includes remote mountainous areas making travel to treatment sites a time consuming and potentially dangerous patient endeavor, especially during winter months. Travel time for medical care is also a significant contributor in limiting patient access to VA services in many other regions of the country. These remote areas, where approximately $40 \%$ of VA's 8 million enrollees live [5], are frequently lower income areas and financial burdens related to travel expenses and time also play a part in restricting access to services. Additional barriers to accessing care for patients in remote areas include lack of information or local services, and lack of care coordination [6].

While the CBOCs serve to alleviate some of the barriers to timely treatment for patients, it may not be feasible to establish long-term comprehensive, specialized rehabilitation services in every remote community. Additionally, the CBOCs also face barriers to comprehensive patient care service through facility size and staffing level constraints. In some instances, patients may particularly benefit from ongoing access to specialist care facilitated through telerehabilitation [7].

Telerehabilitation is a branch of telemedicine that focuses on the utilization of advanced and reliable telecommunication strategies to provide distance support, rehabilitation services, and information exchange between individuals with disabilities and their clinical providers [8]. It can be used in consultation with providers who treated the patient in the hospital, thereby improving coordination and continuation of care. Home health nurses and therapists can utilize telerehabilitation to obtain important information prior to home visits, thereby increasing efficiency and efficacy. In an effort to reach as many patients as possible, the VA Clinical Video Telehealth (CVT) program was designed and established to improve access for patients in remote area CBOCs through video conferencing as an aid to treatment services [9].

In recent years, rapid technological advances in personal communication devices have provided new opportunities for their use to provide care, education and support to patients and families in remote locations. Studies proposing use of the rapidly advancing consumer personal communication technologies to facilitate remote patient care through Internet-based remote video evaluation have been conducted with stroke patients [10], but not with the polytrauma population. More importantly, Bergquist, et al. [11] demonstrated that patients with acquired brain injury and memory impairment can learn how to successfully use an internet-based cognitive rehabilitation program. Additional studies indicate that individuals with brain injury are interested in accessing telerehabilitation services [12-13]. However, there is minimal data regarding whether these patients have access to the necessary telecommunication devices. As new communication technologies develop, we need a clear understanding of the acceptance of those technologies by the intended service population. Our unique access to the TBI out-patient population allows this study to fill the knowledge gap by exploring the availability of advanced telecommunication devices to PNS outpatients, living greater than 60 miles from our site. 


\section{Materials And Methods}

\section{Participants}

Three hundred and fifty-five unique patients were seen in the Polytrauma Network Site (PNS) outpatient clinic at the VA Palo Alto Healthcare System from June, 2009 - May, 2010. From this cohort, prospective study inclusion criterion was living greater than 60 miles from the VHA, Palo Alto. Additionally, to participate in the survey, patients had to respond to one of three attempts at phone contact during the seven-month period of study enrollment from June 2010 to December 2010.

\section{Survey instrument and procedure}

All necessary IRB and local VA Research agency approvals were obtained prior to commencement of any project activities. Participants were surveyed individually via telephone during an interview requiring less than 60 minutes to complete. All participants provided informed verbal consent prior to answering any survey questions. The survey instrument was designed to ascertain respondents' ownership and utilization of various advanced means of communication. As this was a technological capability survey, no questions regarding respondents' willingness to engage in remote Telehealth programs were asked.

To determine the extent of cell phone utilization and the capabilities of the cell phones being used by this group of patients, respondents were asked a number of questions regarding ownership, the types of cell phones, and the frequency of cell phone use. We specifically inquired as to whether individuals had Smartphones, other cell phones with text messaging features, or more basic cell phones with only voice communication capabilities. Smartphones are basically the convergence of mobile computers and cellular telephones, with the capacity to transmit voice, text and multimedia messages, as well as provide computing power and internet capabilities [14].

Surveys were administered in a structured format, with all responses recorded by the interviewer. Both yes/no and open-ended questions were included. Responses to open-ended questions were recorded verbatim, and were probed as necessary to obtain specific relevant information.

\section{Data analysis}

The data was summarized with basic descriptive methods such as cross-tabulations for frequencies and proportions of responses using Microsoft Access. Qualitative analyses were used for the data consisting of brief text entries (i.e. responses to open ended questions).

\section{Results \\ Demographics}

Of the 159 patients that met our inclusion criteria, we were able to contact 82 and unable to reach the remaining 77 as the telephones repeatedly went to voicemail service and calls were not returned. Ultimately, 70 patients completed the survey, which is an overall response rate of $44 \%$ (Figure 1). 


\section{Cureus}

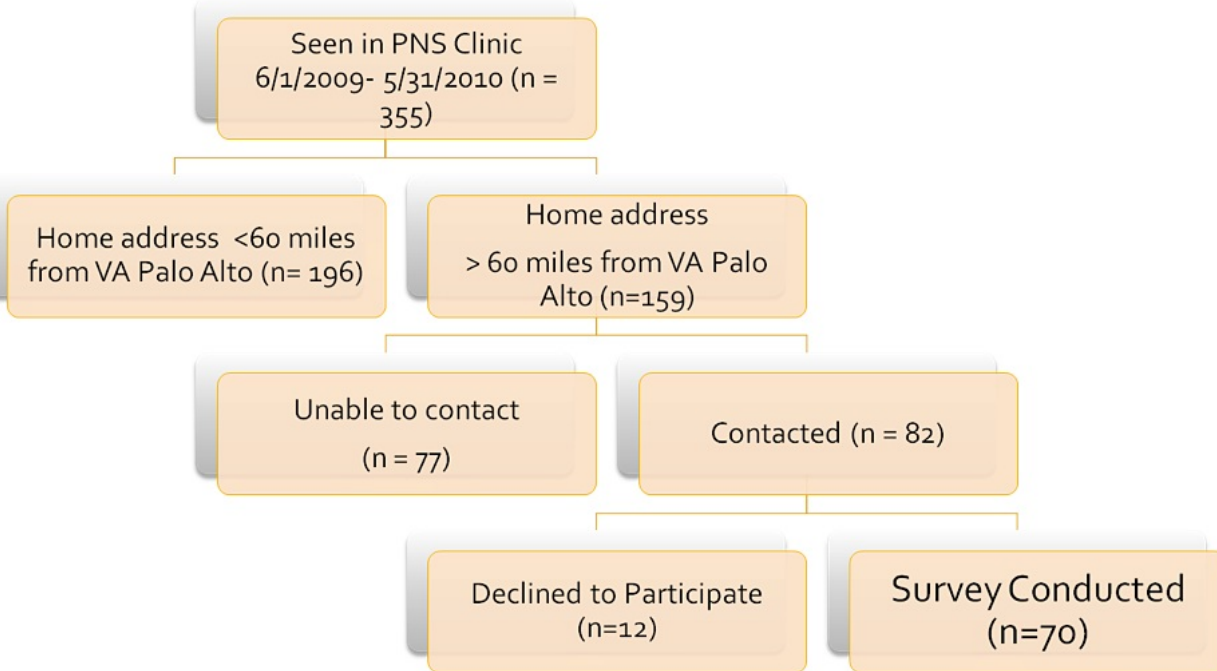

FIGURE 1: Design and Study Flowchart

Ninety-one percent of the respondents were male, with the remaining $9 \%$ female. Seventy-six percent were between $20-39$ years old, with $24 \%$ from $40-79$ years old. The majority of respondents were Veterans (82\%), with the remaining respondents serving as Active Duty (4\%), National Guard (7\%), or Reservists (7\%). The majority of respondents were Caucasian (67\%; Figure 2).
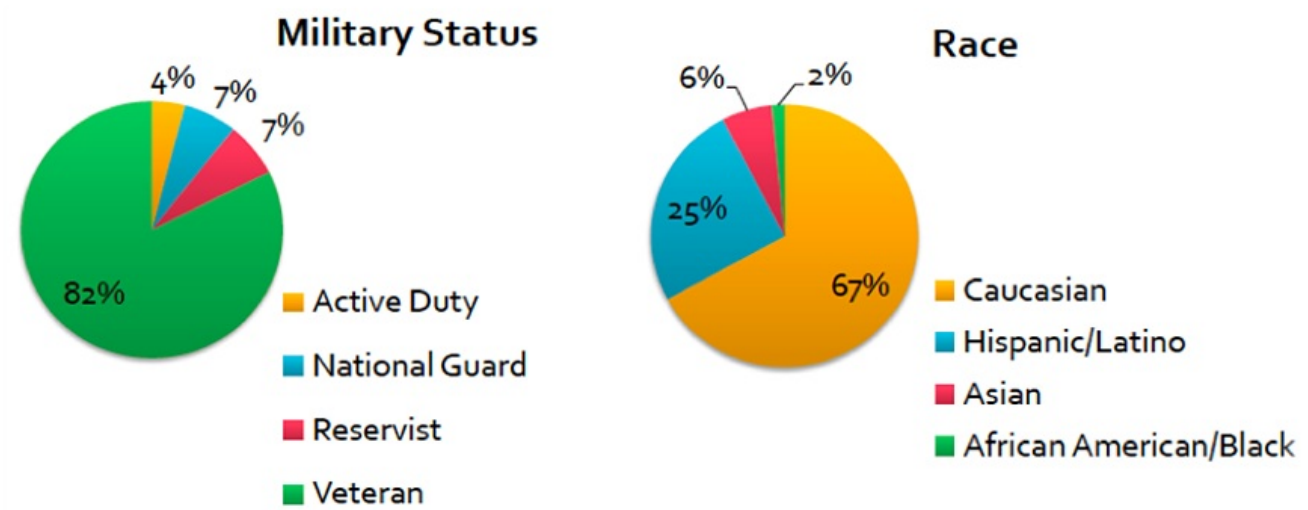

FIGURE 2: Military status and Ethnicity

\section{Landline telephone utilization}

Forty percent (28/70) of survey respondents reported having a landline telephone in their homes. Of those, only $21 \%(6 / 28)$ used it daily, while $54 \%(15 / 28)$ used it at least once weekly. The remaining $25 \%(7 / 28)$ used it less often than once monthly. In response to the open ended question of why individuals did not have a land line, 62\% (26/42) indicated a landline was unnecessary because they used their cell phone instead, 28\% (12/42) said they did not need one because they were not home enough for it to be useful, and 10\% (4/42) indicated cost as the 
primary reason for non-ownership.

\section{Cell phone ownership and utilization}

Our surveys indicated that $94 \%$ (66/70) of the respondents own and use a cell phone. Forty-four percent (29/66) of these cell phones were Smartphones, with over half of these $(52 \%, 15 / 29)$ being the Apple iPhone. Among the individuals that had a cell phone other than a Smartphone, $86 \%(32 / 37)$ had a data plan such as text messaging.

\section{Computers ownership and utilization}

The majority of respondents $(93 \%, 65 / 70)$ owned and used a working computer at their residence (Figure 3). Among those respondents who currently have and use a computer, $86 \%$ $(56 / 65)$ reported having a Windows operating system, while $10 \%(7 / 65)$ had a Macintosh. One (2\%) reported having both a Macintosh and a Windows system, and another (2\%) had both a Windows and a Linux system.

\section{Computer Use}

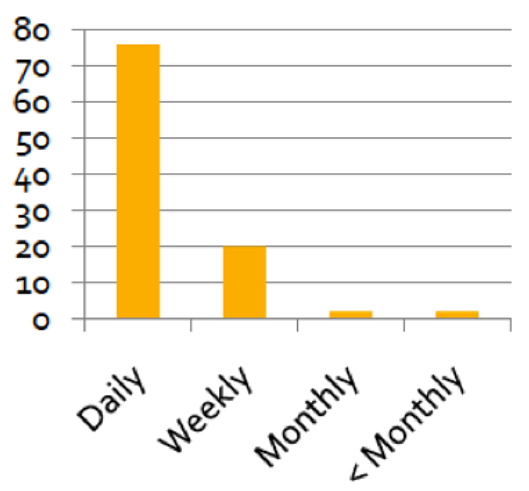

Internet Use

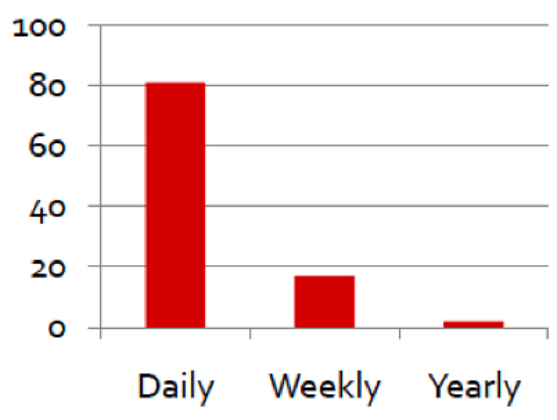

FIGURE 3: Computer and Internet Use

To further assess exposure to computer technology, respondents were asked how often they typically use any computer (at home, work, school, library, etc). Seventy-seven percent (50/65) of respondents reported using a computer daily, 20\% (13/65) used a computer weekly, and 3\% $(2 / 65)$ used it less than weekly.

\section{Internet usage}

Ninety-three percent (65/70) of the total survey respondents used the Internet on either a computer or a Smartphone, or both. Specifically, $89 \%(62 / 70)$ reported using the Internet on their computer, while $43 \%$ (41/70) used the Internet on their cell phone. Eighty-two percent $(53 / 65)$ of all Internet users reported at least daily use, and $17 \%(11 / 65)$ indicated at least weekly use. One individual indicated using the Internet a few times per year.

The type of Internet connections that individuals use is an important consideration with regard to information security. Of those individuals who use the Internet on a computer, 55\% (34/62) use only wireless connection, 24\% (15/62) used an Ethernet cable, and 21\% (13/62) use both wireless and Ethernet. Of those who use wireless Internet, 94\% (44/47) connect via a personal password-protected wireless network, while the remaining 6\% (3/47) of individuals used a shared network. 
The small percentage of individuals who do not own or use computers and/or Internet (7\%, 5/70) cited cost as the primary deterrent.

\section{Cost of communication technology services}

The average monthly cell phone cost was $\$ 80$, whereas the average monthly landline phone cost was $\$ 19$. Approximately $78 \%$ of landline phones were bundled with internet and/or cable television services, with an average cost of internet/phone bundle of $\$ 71$ and an average cost of internet/phone/cable bundle of $\$ 123$. The average monthly cost of internet services only was \$46 (Figure 4).

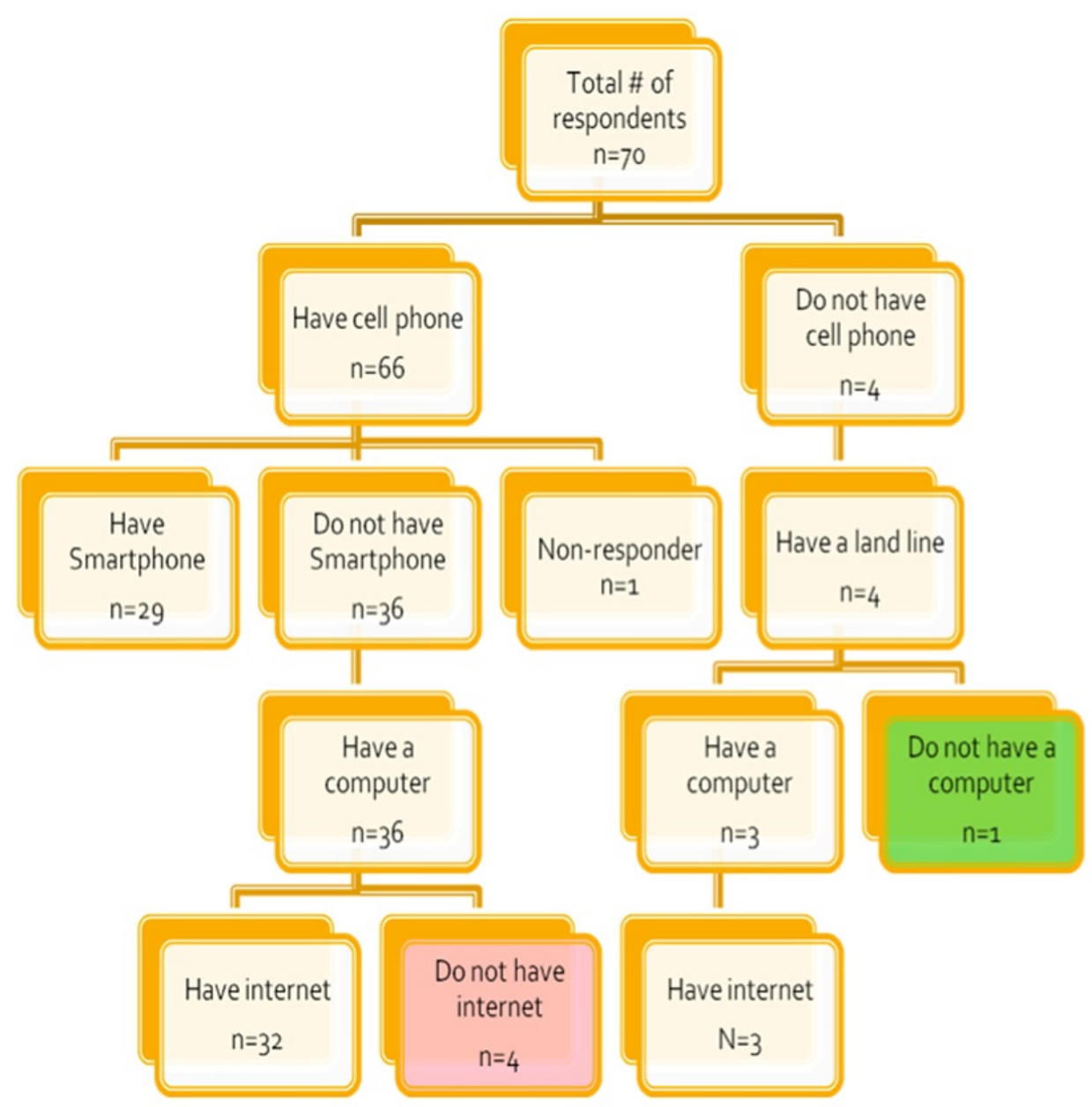

FIGURE 4: Summary of Current Resources Utilized by Study Participants

\section{Discussion}

The PNS was established to address the needs of polytrauma outpatients over a wide geographic area and consistently serves over 350 patients per year. Of these, $45 \%$ live greater than 60 miles from the service site and could benefit from remote access to their health care due to the distances, cost, and road and traffic issues associated with travel in our geographic area. Telerehabilitation can increase access to post-acute rehabilitation services by reducing the need for travel between patients' homes and the rehabilitation clinic, a particular benefit for patients isolated by disability, poverty, or geography. This is highly relevant to the Veterans Health 
Administration (VHA), where approximately $40 \%$ of its 8 million enrollees live in remote areas $[5]$.

The VA has responded to this demographic by establishing the CVT program to improve access for patients in remote area CBOCs through video conferencing. However, this program still entails a level of travel to receive treatment services. Studies have shown that there is great interest in the possibility of accessing telerehabilitation services via in home internet technology among individuals in this patient population. A key concern is extending these services to specific populations, such as those with TBI due to potential cognitive impairments. Previous work has established the validity of telephone surveys in this population and demonstrated that given the appropriate assistive technologies, even individuals with significant brain trauma can engage with distant providers via the Internet.

Despite these promising findings, there is no data available regarding whether patients with TBI have access to the technological devices, such as home computers or internet capable mobile devices necessary to utilize web-based telerehabilitation interventions.

We surveyed $44 \%$ of a cohort representative of the larger remote polytrauma outpatient population served by our site regarding their use of various communication devices. In this cohort, knowledge and utilization of new technologies were relatively high for ownership and regular use of a Smartphone, ownership and use of a computer, and daily Internet use.

Certain potential limitations inherent in the study need to be acknowledged. Notably, respondents were recruited from a convenience sample of patients connected with a polytrauma outpatient clinic. Thus, this sample cannot be assumed to be representative of the TBI population overall, but rather is specific, by design, to focus on those in remote areas.

The survey content (telecommunication devices use and accessibility) and response rate are also important considerations. Persons familiar with advanced communication technologies may be more inclined to participate in this type of study, while individuals not necessarily interested in this topic may be less likely to respond. Although the $44 \%$ response rate in this study is relatively high considering the nature of survey studies, we were unable to contact nearly half of the individuals who met the criteria for inclusion in the study via telephone. This is a concern since any telephone-based intervention would require that patients be readily contacted via telephone. However, this does not exclude the possibility that these individuals could be contacted through alternate communication strategies available, such as Internet or cell phone.

Future studies need to be conducted to determine the characteristics of those individuals we were unable to reach, as well as to investigate alternative strategies for telerehabilitation in this subset of patients.

It is also important to recognize that this study did not address the efficacy of telephone and internet-based approaches for telerehabilitation, but rather assessed patients' technological capabilities in order to establish a foundation of feasibility for deployment of web based telerehabilitation programs through emerging technologies. Individuals with less education and/or technical experience may find it difficult to utilize newer technology, particularly in the presence of cognitive dysfunction [12]. Thus, the efficacy of utilizing telerehabilitation strategies versus traditional modalities of care in this patient population need to be further examined.

\section{Conclusions}


Despite the inherent caveats, some important observations can be drawn from the present study. Almost half the respondents owned and used a Smartphone, including the internet options available on these devices. Among patients who did not have a Smartphone, the majority owned a cell phone and a computer with Internet access, thus possessing equivalent, but less mobile, web access.

In total, our data suggests that a significant proportion of individuals with TBI living in remote areas have access to advanced communication devices that may potentially be utilized to facilitate their care through web based telerehabilitation. This information can be used as a foundation for design of future studies geared at implementation and management of these technologies.

\section{Additional Information}

\section{Disclosures}

Human subjects: All authors have confirmed that this study did not involve human participants or tissue. Animal subjects: All authors have confirmed that this study did not involve animal subjects or tissue. Conflicts of interest: In compliance with the ICMJE uniform disclosure form, all authors declare the following: Payment/services info: All authors have declared that no financial support was received from any organization for the submitted work. Financial relationships: All authors have declared that they have no financial relationships at present or within the previous three years with any organizations that might have an interest in the submitted work. Other relationships: The data collection for this project was deemed to NOT be human subject research by the Stanford IRB Research Compliance Office on June 2, 2010.

\section{Acknowledgements}

We would like to thank Dr. Jeffery Teraoka and the VA Palo Alto Polytrauma Network Site staff, particularly Rose Salerno, RN, as well the research staff at the VA Palo Alto Defense Veterans Brain Injury Center for their assistance in conducting this study. The views expressed in this article are those of the authors and do not necessarily represent the views of the Department of Defense (DOD) or the Veterans Health Administrations (VHA). The data collection for this project was deemed to NOT be human subject research by the Stanford IRB Research Compliance Office on June 2, 2010. All subject contact was via telephone and participants gave verbal consent to proceed with the survey.

\section{References}

1. Bell KR, et al: The effect of telephone counselling on reducing post-traumatic symptoms after mild traumatic brain injury: a randomised trial. J Neurol Neurosurg Psychiatry. 2008, 79:12751281. 10.1136/jnnp.2007.141762

2. Okie S: Traumatic brain injury in the war zone . N Engl J Med. 2005, 352:2043-2047. 10.1056/NEJMp058102

3. Numbers for Traumatic Brain Injury. (2011). Accessed: Feb 10, 2011: http://www.dvbic.org/dod-worldwide-numbers-tbi.

4. Warden D: Military TBI during the Iraq and Afghanistan wars . J Head Trauma Rehabil. 2006, 21:398-402. 10.1097/00001199-200609000-00004

5. Darkins, A, et al: Care Coordination/Home Telehealth: the systematic implementation of health informatics, home telehealth, and disease management to support the care of veteran patients with chronic conditions. Telemed J E Health. 2008, 14:1118-1126. 10.1089/tmj.2008.0021

6. Sample PL, Darragh AR: Perceptions of care access: the experience of rural and urban women following brain injury. Brain Inj. 1998, 12:855-874. 10.1080/026990598122089

7. Darkins A, et al: Enhancing access of combat-wounded veterans to specialist rehabilitation 


\section{Cureus}

services: the VA Polytrauma Telehealth Network. Arch Phys Med Rehabil. 2008, 89:182-187. 10.1016/j.apmr.2007.07.027

8. Chumbler NR, et al: Study protocol: home-based telehealth stroke care: a randomized trial for veterans. Trials. 2010, 11:74. 10.1186/1745-6215-11-74

9. Clinic Based Telehealth Operations Manual . (2012). Accessed: July, 2012: http://199.237.254.34/pubs/pdf/Telehealth.pdf.

10. Meyer BC, et al: Prospective reliability of the STRokE DOC wireless/site independent telemedicine system. Neurology. 2005, 64:1058-1060. 10.1212/01.WNL.0000154601.26653.E7

11. Bergquist $\mathrm{T}$, et al: Internet-based cognitive rehabilitation in individuals with acquired brain injury: a pilot feasibility study. Brain Inj. 2008, 22:891-897. 10.1080/02699050802405487

12. Ricker JH, et al: Telerehabilitation needs: a survey of persons with acquired brain injury . J Head Trauma Rehabil. 2002, 17:242-250. 10.1097/00001199-200206000-00005

13. Hart T, Buchhofer R, Vaccaro M: Portable electronic devices as memory and organizational aids after traumatic brain injury: a consumer survey study. J Head Trauma Rehabil. 2004, 19:351-365.

14. Busis N: Mobile phones to improve the practice of neurology . Neurol Clin. 2010, 28:395-410. 10.1016/j.ncl.2009.11.001 